\title{
NÃO EXISTE ESTRUTURA RELATIVA EM CHINÊS?
}

\author{
THERE ARE NO RELATIVE CONSTRUCTIONS IN CHINESE?
}

Chunyuan Wang ${ }^{1}$

\begin{abstract}
RESUMO: A gramática tradicional propõe que não existe, como há em português e em inglês, a estrutura relativa em chinês. O que existe seria uma estrutura atributiva (attributive structure) em que a oração relativa seria um modificador que ficasse anterior do núcleo para modificá-lo. Por outro lado, estudo da estrutura relativa sempre é um assunto interessante e cheio de divergências no modelo gerativo. Alguns linguistas propõem que a estrutura relativa em chinês é resultado de movimento de Op, como o caso de Ning (1993), alguns propõem que é resultado de movimento de núcleo DP como o caso de Wang (2005) e outros propõem que algumas relativas são resultado de movimento de Op e outras são resultado de movimento do núcleo NP como o caso de Aoun\&Li (2003). Contudo, esses estudos raramente examinam as evidências de avaliação propostas por Chomsky (1977, 1981), como Reconstrução, Subjacência/efeitos de ilha e Parasitic gap para examinar se uma estrutura relativa em chinês é resultado de movimento. Esse estudo tem por objetivo, tendo em vista preencher essa lacuna, examinar as evidências contra os dados linguísticos concretos em chinês para corroborar que existe estrutura relativa em chinês que é uma estrutura atributiva verbal tratada por gramática tradicional e é resultado de movimento.
\end{abstract}

Palavras-chave: Estrutura relativa em chinês; estrutura atributiva em chinês; reconstrução; subjacência/efeitos de ilha; Parasitic gap.

ABSTRACT: Grammarians of traditional grammar propose that there is no, as there is in Portuguese and English, relative structure in Chinese. What exists would be an attributive structure in which the relative clause would be a modifier that stays before the head to modify it. However, the study of relative structure is always an interesting subject and full of divergences in the generative model. Some linguists propose that relative structure in Chinese is the result of Op movement, such as the case of Ning (1993), some propose that it is the result of DP head movement, as the case of Wang (2005), and others propose that some relatives are result of Op movement and others are result of NP head movement, as the case of Aoun

\&Li (2003). Nevertheless, these studies rarely examine the evaluation evidences proposed by Chomsky (1977, 1981), such as Reconstruction, Subjacency/island effects, and Parasitic Gap to examine whether a relative structure in Chinese is the result of movement. This study aims to fill this gap by examining these evidences against concrete language data in Chinese, in order to corroborate that there is relative structure in Chinese and it is the result of movement.

Keywords: Relative structure in Chinese; attributive structure in Chinese; reconstruction; subjacency/island effects; Parasitic gap.

\section{Estrutura modificador-núcleo na gramática tradicional}

A estrutura modificador-núcleo ${ }^{2}$ (Modifier-head structure) em chinês apresenta estado

\footnotetext{
${ }^{1}$ Mestrando em Linguística e Letras na Universidade Católica do Rio Grande do Sul-PUC/RS. Bolsista CAPES.
} 
sintático especial diferente das demais estruturas, no que diz respeito à sua função sintática e categorização. Ela é classificada em estruturas modificador-núcleo nominal, adjetival e verbal de acordo com a função do núcleo de palavra nocional (notional word head) que as contém, como mostram (1), (2) e (3). A estrutura modificador-núcleo nominal se destaca entre as três estruturas, e é essa estrutura considerada por muitos linguistas como uma estrutura relativa, contrária à proposta dos gramáticos que a consideram uma estrutura modificador-núcleo.
(1) gongsi lingdao
(nominal) empressa chefe
(2) feichang piaoliang (adjetival) muito bonito
(3) renzhen gongzuo (verbal)
dedicadamente trabalhar

Essas estruturas modificador-núcleo são categorizadas também em prototípicas e atípicas, além da sua classificação sintática acima apresentada. Elas são tratadas como se fossem palavras nocionais que têm suas próprias funções sintáticas. Estrutura prototípica e atípica se diferem em a capacidade de subcategorizar. Estrutura prototípica é subcategorizada em estrutura atributiva (attributive structure) e estrutura adverbial (adverbial structure). A incapacidade de se subcategorizar da estrutura atípica se deve ao fato da opacidade de tipo e domínio dos constituintes (modificador e núcleo) da estrutura, entre outros. Obviamente, exaustar os motivos dessa incapacidade não é nossa intenção, leitores interessados podem consultar Zhang\&Han (1997) para mais detalhes.

A estrutura atributiva é composta de sintagma atributivo e núcleo nominal e sua função sintática é nominal. Essa função sintática é determinada pelo tipo do seu núcleo. Por isso, a função sintática da estrutura atributiva é nominal e a da estrutura adverbial é verbal ou adjetival, como pode se ver a seguir. Sintagma atributivo consiste de adjetivo, substantivo e sintagma verbal ${ }^{3}$ como mostram (4), (5) e (6). Pode-se observar que quando a sintagma atributivo for adjetivo ou substantivo, de é apagável como mostram (4) e (5). E quando ele for sintagma verbal (CP), de não é apagável como mostra (6). Este estudo propõe que, a partir desta observação, que estrutura atributiva verbal é de fato uma estrutura modificador-núcleo falsa. Ela é estrutura relativa, na medida em que apresenta a não apagabilidade de de que é na verdade um $\mathrm{C}$, o que é a característica essencial da estrutura relativa em chinês (7a), diferente do da estrutura relativa em inglês na qual a ausência de C não causa a gramaticalidade, como mostra

\footnotetext{
${ }^{2}$ Uma das evidências dos gramáticos da gramática tradicional de proporem não existir estrutura relativa em chinês é o fato de que o modificador que descreve núcleo fica anterior do núcleo, como mostra (a), enquanto modificador fica posterior do núcleo nas línguas como inglês e português, como mostra (b).

(a) wo ai de [Head nvhai] Eu amar que menina

(b) [Head A menina] que eu amo [Head The girl] who I love

${ }^{3}$ Sendo esse sintagma verbal chamado de CP, no modelo gerativo, que fica posterior do núcleo e o modifica nas línguas como português e inglês, como mostra a nota de rodapé $1 \mathrm{~b}$ e que fica anterior do núcleo e o modifica nas línguas como chinês, como mostra a nota de rodapé 1a.
} 
(7b), enquanto as estruturas atributivas tradicionais de adjetivo e de substantivo apresentam a apagabilidade de de. Essa diferença de de apagável ou não apagável determina que a estrutura atributiva verbal é estrutura relativa enquanto as tradicionais de adjetivo e de substantivo são estruturas modificador-núcleo verdadeiras.

(4) meili $(d e)^{4}$ chuanshuo
linda lenda
(5) gongsi $(d e)^{5}$ lingdao
empresa chefe
(6) wo ai de nvhai
eu amar que menina
(7) a. ${ }^{*}$ [ [CP wo ai $\mathrm{C}^{0}$ ] [NP nvhai]
[CP wo ai de] [NP nvhai]
b. [NP the girl] [CP $\mathrm{C}^{0} /$ that/who I like]

A estrutura adverbial é composta de advérbios ou adjetivo de estado (state adjective) e núcleo verbal ou adjetival. Sua função sintática é verbal ou adjetival. Como a estrutura adverbial é muito diferente da estrutura relativa e é impossível considerá-la como estrutura relativa, este estudo escolhe não detalhá-la, leitores interessados podem se dirigir a Zhou (2009) para a referência.

Em resumo, essas estruturas modificador-núcleo são especiais em termos de sua função sintática idêntica ao seu núcleo e de sua categorização como prototípica e atípica, por um lado, os gramáticos da gramática tradicional tem razão para propor que não existe estrutura relativa em chinês, na medida em que, em primeiro lugar, o fato de modificador (CP) ficar anterior do núcleo é muito diferente para línguas como inglês e português em que CP fica posterior do núcleo; em segundo, existem estruturas atributivas tradicionais e típicas de substantivo e de adjetivo que permitem a apagabilidade de de, por outro. Porém, existe também a estrutura atributiva verbal que não permite apagar de, o que a deixa especial e não parece uma estrutura atributiva real, ou seja, ela é de fato uma estrutura relativa.

\section{Estrutura Relativa na Gramática Gerativa}

Estrutura relativa (relative construction) é uma estrutura nominal composta por oração relativa (relative clause) e núcleo nominal (head noun). Na análise do núcleo externalizado (The Head External Analysis) de Chomsky (1977), a oração relativa é um CP que tem seu núcleo C anteriorizado (head-initial C), como (8) e (9) mostram em inglês e em português. Ning (1993) propõe que "de" é um núcleo $\mathrm{C}$ e que ele é posteriorizado (head-final $\mathrm{C}$ ) em relalção ao IP, como mostra (10), contrário ao C na relativização em inglês, que é anteiorizado. Além disso, outra diferença marcante entre inglês, português e chinês é a posição do núcleo da estrutura

\footnotetext{
${ }^{4}$ Quando o sintagma atributivo for adjetivo, "de" é apagável.

${ }^{5}$ Quando o sintagma atributivo for substantivo, "de” é apagável.
} 
relativa. Vries (2002) classifica sintaticamente quatro tipos pricinpais de estruturas relativas, a saber, estrutura relativa pós-nominal (postnominal relatives), estrutura relativa prenominal (prenominal relatives), estrutura relativa circumnominal (circumnominal relatives) e estrutura correlativa (correletives). Segundo Vries (2002), entre as línguas com ordem SVO, inglês e português pertencem à estrutura relativa com núcleo anteriorizado enquanto chinês pertence à com núcleo posteriorizado, como mostram (8), (9) e (10).
(8) [Head the girl] [CP C that [IP I love]]
(pós-nominal)
(9) [Head aquela moça] [CP C que [IP eu amo]]
(10) [ [CP [IP wo ai] ${ }_{\mathrm{C}}$ de] $]$ [Head $_{\text {nage nvhai] }}$
(prenominal)
Eu amo que aquela moça

...Their syntactic structures are sketched in (23).
(23) a. postnominal relatives
$[$ s.matrix $\ldots . . \mathrm{N} \mathrm{RC]} \mathrm{...} \mathrm{]}$
b. prenominal relatives
$[s$-matrix $\ldots . .[\mathrm{RC} \mathrm{N}] \ldots$.
c. circumnominal relatives
d. correlatives
$\left[s\right.$-matrix $\ldots\left[\left[\left[_{R C} \ldots . . . . ..\right]\right] \ldots.\right]$
$\left[s_{\text {-matrix }}\left[{ }_{R C}(\ldots) \mathrm{N} \ldots\right]\left[\left[_{\text {S-matrix }} \ldots(\right.\right.\right.$ Dem) $\ldots]$

(VRIES, 2002, P. 20)

Em geral, são três análises de movimento para a derivação da estrutura relativa, a saber, a análise do núcleo externalizado proposta por Chomsky (1977), a análise de promoção do núcleo (The Head Raising Analysis) proposta por Schachter (1973), Kayne (1994) e Vries (2002) e a análise eclética (The Matching Analysis) proposta por Aoun\&Li (2003). Entre as quais, a oração relativa é considerada como adjunto do núcleo na análise do núcleo externalizado como mostra (11a) e (12b), como complemento de $\mathrm{D}^{6}$ na análise de promoção do núcleo como mostra nota de rodapé (5b) aqui repetida (12a), (12b) e (12c) e como complemento de D para estrutura relativa em inglês, como mostra (13a), (13b) e (13c), na análise eclética.

(11) a. [DP the [NP girl $\left._{\mathrm{i}}\right]$ [CP who $_{\mathrm{i}}$ I like $\left.\left.\left.\mathrm{t}_{\mathrm{i}}\right]\right]\right]$

b. [DP the [NP $\left.\operatorname{girl}_{\mathrm{i}}\right]$ [CP $_{\mathrm{CP}} \mathrm{p}_{\mathrm{i}}$ (that) I like $\left.\left.\left.\mathrm{t}_{\mathrm{i}}\right]\right]\right]$

(12) a. [DP the [CP [NP girl $]_{\mathrm{i}}\left[\right.$ (that) I like $\left.\left.\left.\mathrm{t}_{\mathrm{i}}\right]\right]\right]$

b. [DP the [CP [DP [NP girl $]_{j}$ which $\left.t_{j}\right]_{k}\left[C^{0}\right.$ I like $\left.\left.\left.t_{k}\right]\right]\right]$

c. [DP the [CP [DP [NP girl $\left.]_{j} O p t_{j}\right]_{k}\left[\right.$ that I like $\left.\left.\left.t_{k}\right]\right]\right]$

(13) a. [DP a [ForceP [NP Maria $]_{i}\left[\right.$ ForceP que [TopP $t_{i}\left[T_{\text {Topo }}\right.$ [IP o João ama $\left.\left.\left.\left.\left.t_{i}\right]\right]\right]\right]\right]$

b. [DP a [Forcep [NP Maria] [Forcep $\mathrm{F}^{0}$ [Topp quem [Top $^{0}$ [IP o João ama $\left.t_{\mathrm{i}}\right]$ ]]]]]

c. [DP uma [ForceP [NP menina] [Forcep que [TopP $\mathrm{Op}_{\mathrm{i}}\left[\mathrm{Top}^{0}\right.$ [IP o João ama $\left.\left.\left.\left.\left.\mathrm{t}_{\mathrm{i}}\right]\right]\right]\right]\right]$

\footnotetext{
${ }^{6} \mathrm{Na}$ fase inicial da análise de promoção do núcleo, Schachter (1973) propõe que a oração relativa CP é adjunto do núcleo como mostra (a) a seguir. Somente a partir da posposta de Teoria de Assimetria de Kayne (1994), a oração relativa é proposta como complemento de D como mostra (b).

(a) [nP a menina $]_{i}\left[\right.$ CP que [ip o João ama $\left.\left.\mathrm{t}_{\mathrm{i}}\right]\right]$

(b) [DP the [CP [NP girl $]_{\mathrm{i}}\left[(\right.$ that $)$ I love $\left.\left.\left.\mathrm{t}_{\mathrm{i}}\right]\right]\right]$ [DP the [CP [DP [NP girl $]_{j}$ which $\left.t_{j}\right]_{k}\left[C^{0}\right.$ I like $\left.\left.\left.t_{k}\right]\right]\right]$ [DP the [CP [DP [NP girl $]_{j}$ Op t $]_{\mathrm{j}}\left[\right.$ that I like $\left.\left.\mathrm{t}_{\mathrm{k}}\right]\right]$ ]
} 


\subsection{Análise do núcleo externalizado}

$\mathrm{Na}$ análise do núcleo externalizado, o operador wh- ou operador vazio $(\mathrm{Op})$ se move para a posição [Spec-CP] deixando o vestígio de wh- (variável, Vbl). O núcleo é gerado na base (basegenerated) que fica externalizado da oração relativa CP. Aoun \&Li (2003) resume a derivação dessa análise como mostra (14). Segundo essa análise, a derivação de (11a) repetida aqui como (15a) com movimento de operador se deve ao fato de que o operador "who" se move da posição de objeto para [Spec-CP] deixando vestígio Vbl t, por um lado, a de (11b) repetida aqui como (15b) com movimento de Op é resultado de Op se mover da posição de objeto para [Spec-CP] deixando vestígio $\mathrm{Op}$ t.

(14) $\quad\left[\mathrm{NP} / \mathrm{DP}\left[\text { Head } \mathrm{NP} / \mathrm{DP}_{\mathrm{i}} \ldots . .\right]\left[\text { Relative CP } w h_{\mathrm{i}}\left[\mathrm{IP} \ldots \mathrm{t}_{\mathrm{i}} \ldots .\right]\right]\right]^{7}$ (AOUN \&LI, 2003, P100)

(15) a. [DP the [NP girl $\left.\mathrm{i}_{\mathrm{i}}\right]$ [CP who $\mathrm{I}$ like $\left.\left.\left.\mathrm{t}_{\mathrm{i}}\right]\right]\right]$ b. [DP the [NP girl $\mathrm{i}_{\mathrm{i}}\left[_{\mathrm{CP}} \mathrm{Op}_{\mathrm{i}}\right.$ (that) I like $\left.\left.\mathrm{t}_{\mathrm{i}}\right]\right]$

Segundo essa análise, o processo de movimento faz com que se estabeleça a cadeia (wh,Vbl) formulando assim a relação de co-indexação entre núcleo, operador wh- e vestígio Vbl, através de operação de reconstrução. Em (16), haveria uma co-indexação entre the Picture of herself, which e vestígio Vbl t, e herself do núcleo se referiria à Maria do CP se tudo desse correto. Porém, pode se observar que Maria não pode reger herself, na medida em que o estado sintático do CP é um adjunto. Se Maria regesse herself ultrapassando CP, seria desobedecido o princípio A proposto por Chomsky (1981). Para satisfazer esse princípio, o conceito de reconstrução é proposto. O núcleo DP é reconstruído para a posição de $t_{i}$ de onde operador wh- se moveu como mostra (17). Mesmo que a derivação fique agora gramatical sintaticamente, essa análise implicaria, em primeiro lugar, ter dois movimentos, a saber, movimento de operador e reconstrução do núcleo, o que desobedece o princípio de economia, sob a perspectiva mais atual de PM; em segundo, a reconstrução do núcleo ser semelhante à promoção do núcleo que é a ideia da análise de promoção do núcleo. Assim, surgiu a ideia de promoção do núcleo.

(16) [DP the picture of herself $\left.f_{j}\right]_{i}\left[C P\right.$ which ${ }_{i}$ Maria ${ }_{j}$ painted $\left.t_{i}\right]$

(17) $\quad\left[\mathrm{DP} t_{\mathrm{k}}\right]\left[\mathrm{CP}\right.$ which [IP Maria ${ }_{\mathrm{j}}$ painted [DP the Picture of herself $\left.\mathrm{f}_{\mathrm{k}}\right]$ ]

\subsection{Análise de promoção do núcleo}

Kayne (1994) propõe contra à adjunção à direita e sugere que oração relativa CP é complemento do D. Segundo Kayne, NP/DP se move do CP para a posição [Spec-CP] deixando vestígio NP/DP. Segundo essa proposta, (12a) repetida aqui como (18a) é resultado de

\footnotetext{
${ }^{7}$ Vale ressaltar que a análise do núcleo externalizado se madurou com a passagem da fase da Teoria-padrão para Teoria-padrão ampliada revista. Na teoria-padrão, o sintagma nominal é proposto como NP, já na teoria-padrão ampliada revista, ele é proposto como DP.
} 
movimento de NP girl para a posição [Spec-CP] deixando vestígio NP e (12b) repetida aqui como (18b) se dá pelo fato de que, em primeiro lugar, DP which girl se move para a posição de [Spec$\mathrm{CP}]$ deixando vestígio DP; em segundo, NP girl se move para frente do D which. Com base em Kayne (1994), Vries (2002) unifica a análise de movimento NP/DP e propõe que apenas DP que se move do CP para a posição de [Spec-CP] deixando vestígio DP. De acordo com Vries, o movimento de NP seria o de Op+NP na nova proposta. Segundo essa proposta, (18a) seria (12c) aqui repetida como (18c) em que, em primeiro lugar, DP Op girl se move para a posição de [Spec-CP] deixando vestígio DP; em segundo, NP girl se move para frente do D Op.

(18) a. [DP the $\left[\mathrm{CP}[\mathrm{NP} \text { girl }]_{\mathrm{i}}\left[(\right.\right.$ that $)$ I like $\left.\left.\left.\mathrm{t}_{\mathrm{i}}\right]\right]\right]$

b. [DP the [CP [DP [NP girl $]_{j}$ which $\left.t_{j}\right]_{k}\left[C^{0}\right.$ I like $\left.\left.t_{k}\right]\right]$

c. [DP the [CP [DP [NP girl $]_{j}$ Op $\left.t_{j}\right]_{k}\left[\right.$ that I like $\left.\left.\left.t_{k}\right]\right]\right]$

\subsection{Análise eclética}

Segundo Aoun \& Li (2003), a análise de promoção pode explicar bem a derivação da estrutura relativa de argumento, porém, não consegue explicar de forma satisfatória a derivação da de adjunto. Conforme a análise de promoção, na estrutura relativa de argumento, o núcleo é resultado de movimento de argumento da oração relativa como mostra (19), por isso, há uma co-indexação entre núcleo e vestígio. Na estrutura relativa de adjunto, o núcleo dessa estrutura é também um NP, conforme essa lógica, ele deveria ser também resultado de movimento de um NP da oração relativa. Porém, o que se move para essa estrutura é um PP como mostra (20).

(19) A Mariai que o João ama ti

A Maria ${ }_{i}$ que $t_{i}$ ama o João

(20) [Np A caneta] com a qual o João escreve livro t ([pp com a caneta])

A partir dessa observação, Aoun \& Li (2003) propõem que, na estrutura relativa em inglês, estruturas relativas que não contenham operador wh- e que seu determinante seja da "primeira classe" como a (the), essa (this) são resultado de movimento do núcleo como mostra (13a) repetida aqui como (21), e estruturas relativas que contenham operador wh-, bem como as que não o contenham, porém, seu determinante seja da "segunda classe" como algum (some), uma (one) são resultado de movimento de operador wh-/Op como mostram (13b) e (13c) repetida aqui como (22).

(21) [DP a [Forcep [NP Maria $]_{i}\left[\right.$ Forcep que [TopP $t_{i}\left[\operatorname{Top}^{0}\right.$ [IP o João ama $\left.\left.\left.\left.\left.t_{i}\right]\right]\right]\right]\right]$

(22) [DP a [Forcep [NP Maria] [Forcep $\mathrm{F}^{0}$ [Topp quem $_{\mathrm{i}}$ [Top $_{\text {Top }}$ [IP o João ama $\left.\left.\left.\left.\left.\mathrm{t}_{\mathrm{i}}\right]\right]\right]\right]\right]$ [DP uma [Forcep [NP menina] [Forcep que [TopP $O \mathrm{p}_{\mathrm{i}}$ [Top $^{0}$ [IP O João ama $\left.\left.\left.\left.\left.\mathrm{t}_{\mathrm{i}}\right]\right]\right]\right]\right]$

Em resumo, linguistas têm se preocupado com a derivação mais adequada da estrutura relativa, desde as primeiras propostas de Schachter (1973) e Chomsky (1977). Chomsky (1977) propõe que estrutura relativa é resultado de operador wh- ou Op. Porém, quando o núcleo for 
com reflexivo ou for objeto da expressão idiomática (idiom chunk), essa proposta não dá conta de forma satisfatória. Para resolver esse problema, segundo Chomsky (1977), tem que introduzir a operação de reconstrução. Para tratar melhor do problema de reconstrução, Schachter (1973), Kayne (1994) e Vries (2002) propõem a análise de promoção do núcleo para a derivação da estrutura relativa. Realmente, o problema de reconstrução se resolveu com esta proposta. Porém, surgiu um outro problema, Aoun\&Li (2003) observaram que essa proposta não dá conta adequadamente da derivação da estrutura relativa de adjunto e propõem que ela é resultado movimento de PP ou Op, sendo o núcleo gerado na base.

\section{Estudos marcantes para a estrutura relativa em chinês}

\subsection{Ning (1993)}

Ning (1993) divide as orações reltivas em dois grupos, a saber, oração relativa de argumento, aquela em que argumento é relativizao como mostra (23) e oração relativa de adjunto (oração relativa de não-argumento), aquela em que adjunto é relativizado como mostra em (24). Chomsky (1977) propõe que a relativização é ora resultado de moviemnto wh- como mostra (25a) ora resultado de operador vazio (Op) como mostra (25b). O elemento sintático "de" desempenha um papel inportante na relativização em chinês, segundo Ning (1993), esse núcleo $\mathrm{C}$ é posteriorizado em relalção ao IP, contrário ao $\mathrm{C}$ na relativização em inglês. Ning (1993) propõe que, com base em Chomsky (1977), a relativização em chinês é apenas resultado de movimento de $\mathrm{Op}$, dado que não existe pronome relativo wh- em chinês. Ao invés de propor Op na relativização em chinês, Vries (2002) propõe simplesmente que estruturas relativas prenominais apresentam a inexistência do pronome relativo.

(23)a. [NP o livro $]_{i}$ lCP $_{\mathrm{CP}}$ que ele adora $\left.\mathrm{t}_{\mathrm{i}}\right]_{\text {. }}$.

$\left[_{\mathrm{CP}}\right.$ ta xihuan $\left.\left.\mathrm{t}_{\mathrm{i}} \mathrm{de}\right] \mathrm{l}_{\mathrm{NP}} \mathrm{shu}\right]_{\mathrm{i}}$. ele adorar que o livro.

(24)a. $\left[{ }_{\mathrm{NP}} \text { o manual }\right]_{\mathrm{i}}\left[\left[_{\mathrm{CP}}[\mathrm{com} \text { o qual }]_{\mathrm{i}}\right.\right.$ ele concertou o carro $\left.\mathrm{t}_{\mathrm{i}}\right]$.

[CP ta xiu che $\left.t_{\mathrm{i}} d e\right]_{\mathrm{NP}}$ shouce $]_{\mathrm{i}}$.

Ele concertar o carro que o manual.

b. $\left[_{\mathrm{NP}} \text { a garagem }\right]_{i}\left[\left[_{\mathrm{CP}}[\text { onde }]_{i}\right.\right.$ ele concertou o carro $\left.\mathrm{t}_{\mathrm{i}}\right]$.

[CP ta xiu che $t_{\mathrm{i}}$ de] $\left[_{\mathrm{NP}} \text { cheku }\right]_{\mathrm{i}}$.

Ele concertar o carro que a garagem.

(25)a. [NP [NP book $]_{i}\left[{ }_{C P}\right.$ which that $_{\text {IIP }}$ I bought $\left.\mathrm{t}_{\mathrm{i}}\right]$.

b. $\left[_{\mathrm{NP}}\left[\left[_{\mathrm{NP}} \text { book }\right]_{\mathrm{i}}\left[_{\mathrm{CP}} O \mathrm{p}_{\mathrm{i}}\right.\right.\right.$ that $\left[_{\mathrm{IP}}\right.$ I bought $\left.\left.\mathrm{t}_{\mathrm{i}}\right]\right]$.

Three additional properties of prenominal relatives (estrutura relativa prenominal ${ }^{8}$ ) are stated in (39), ...

(39) a. Prenominal relatives do not have relative pronouns.

b. Prenominal relatives do not have clause-initial relative particles.

c. If there is a clause-final relative particle, it does not equal the regular complementizer.

(VRIES, 2002, P. 131)

\footnotetext{
${ }^{8}$ Em relação à definição da estrutura relativa prenominal, refere-se à seção 2 .
} 
Segundo Ning (1993), na relativização de argumento (a), O $\mathrm{p}_{\mathrm{i}}$ se movimenta da posição de objeto para a posição Spec-CP, deixando um vertígio $t_{i}$, o núcleo (shu) da estrutura relativa é gerado na base, sendo a oração relativa CP adjunto do núcleo; da mesma maneira, na relativização de adjunto (b), $\mathrm{Op}_{\mathrm{j}}$ (equivalente ao PP ou adevérbio) se movimenta da posição de adjunto para a posição Spec-CP, deixando um vestígio $t_{j}$, o núcleo (shouce/cheku) da estrutura relativa é gerado na base, sendo a oração relativa $\mathrm{CP}$ adjunto do núcleo.

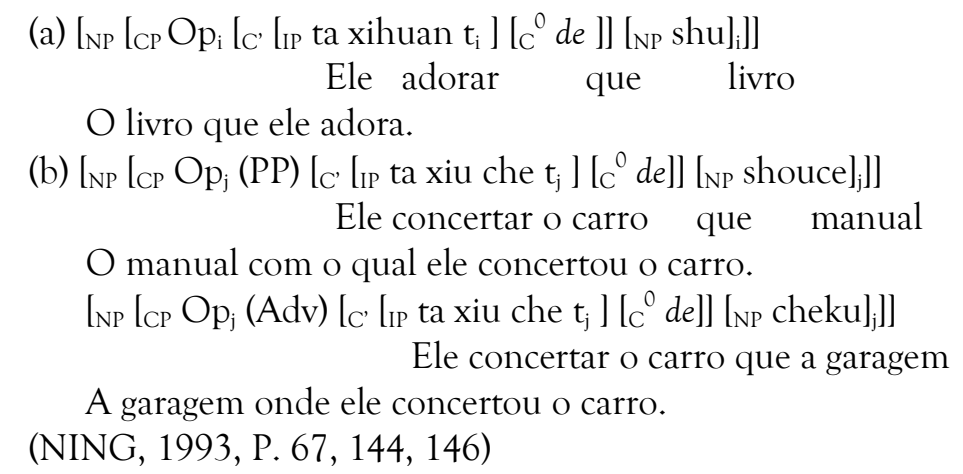

\subsection{AounELLi (2003)}

Diferente da análise para a relativização em inglês para a qual Aoun \& Li (2003) propõe que oração relativa é complemento do determinante, como mostra (13) aqui repetida em (26), não se importando se uma estrutura relativa é resultado de movimento do núcleo ou de operador wh-/Op, Aoun \& Li propõem que, para a análise para a relativização em chinês, a oração relativa é adjunto do núcleo, como mostra (27) e (28), independente dos diferentes tipos de estruturas relativas. A proposta de Aoun \& Li (2003) em relação à análise em chinês vem de Ning (1993), que propõe que, em primeiro lugar, chinês não tem operador como inglês, e, sim, um Op; em segundo, a estrutura relativa em chinês é resultado de movimento de Op sendo oração relativa adjunto do núcleo como propõe Chomsky (1977). Diferente da análise de Ning (1993), Aoun \& Li (2003) propõem que a estrutura relativa de argumento em chinês é resultado de promoção de NP como mostra (27), enquanto a de adjunto é resultado de movimento de Op que equivale ao de PP em inglês, sendo núcleo gerado na base como mostra (28).

The different relative constructions and their properties can be summarized as follows:

(48) NP relativization

a. $\left[\left[_{\mathrm{CP}}\left[\mathrm{IP} \ldots\left[\left[_{\mathrm{NP}} \mathrm{t}_{\mathrm{i}}\right] \ldots\right]\right]\left[_{\text {Head }} \mathrm{NP}\right]_{\mathrm{i}}\right]\right.$

(49) Adjunct relativization $\left[\left[_{\mathrm{CP}} \mathrm{Op}_{\mathrm{i}}\left[\right.\right.\right.$ [IP $\left.\left.\ldots\left[{ }_{\mathrm{PP}} \mathrm{t}_{\mathrm{i}}\right] \ldots.\right]\right][$ Head $\left.\mathrm{NP}]\right]$

(AOUN\&LI, 2003, P. 179)

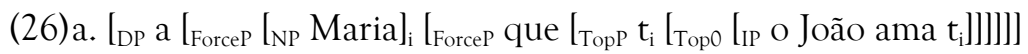

b. [DP a [Forcep [NP Maria] [Forcep $\mathrm{F}^{0}$ [TopP $_{\text {quem }}$ [Top $^{0}$ [IP O João ama $\left.\left.\left.\left.\left.\mathrm{t}_{\mathrm{i}}\right]\right]\right]\right]\right]$

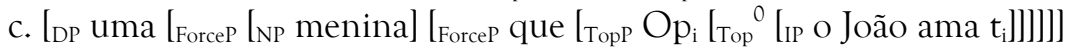

(27) [[CP [IP Lu Xun xie $\left[_{N P} t_{i}\right]$ de $\left.][\text { Head } s h u]_{i}\right]$

Lu Xun escrever $t_{i}$ que livro

(28) [l ${ }_{C P} O p_{k} ~_{I P} L u$ Xun xie shu $\left[_{p P} t_{k}\right]$ de] [Head $\left.\left.b i\right]\right]$ 
$\mathrm{Op}_{\mathrm{k}}$ Lu Xun escrever livro $t_{\mathrm{k}}$ que caneta

Caneta $\left[_{P P} \text { com a qual }\right]_{k}$ Lu Xun escreve livro $t_{k}$

\subsection{Wang (2005)}

A análise de promoção do núcleo vem ocupando uma posição prevalecente para a derivação da estrutura relativa, dado que essa análise trata melhor da explicação de reconstrução de reflexivo e expressão idiomática. Vries (2002) propõe sua análise de promoção do núcleo com base na Teoria de Assimetria de Kayne (1994). Segundo Vries (2002), sua análise trata principalmente da relativização das estruturas relativas restritivas pós-nominais (postnominal restrictive relatives), mas ela pode ser estendida para dar conta da derivação das estruturas relativas prenominais.

The core of my proposal concerning postnominal restrictive relatives is outlined in section 3. Sections 4 through 6 explain how it can be extended to the other syntactic main types of relatives: prenominal, circumnominal and correlative.

(VRIES, 2002, P. 111)

Com base em Vries (2002), Wang (2005) propõe uma análise para a derivação das estruturas relativas em chinês. Segundo Wang (2005), a proposta de Vries (2002) não consegue tratar da relativização em chinês de forma satisfatória, apesar de propor uma derivação precisa, e sujeita à modificação para dar conta disso, na medida em que Vries (2002) adota o Axioma de Correspondência Linear (Linear Correspondence Axiom, LCA) de Kayne (1994), no que diz respeito à ordem liner especificador-núcleo-complementador (Spec-Head-Comp). Segundo Kayne (1994), qualquer ordem linear como, por exemplo, adjução à direita e Comp- Head-Spec, que não obedeça a esse axioma não será licenciada. Para que seja adequadamente licenciada, é preciso fazer uma operação de movimento.

Some linguists have turned to the Minimalist framework to seek a reasonable proposal for the derivation of this construction. Among them, Vries' suggestion is a most attractive one. However, despite his precisely step-by-step derivation, Vries' system cannot accommodate Chinese-type relative clauses. (WANG, 2005, P.12)

Porém, para Wang (2005), não é adequado eliminar a ordem de complemento-núcleoespecificador (Comp-Head-Spec) que possa existir em algumas línguas, na ocasião de ainda não ter uma decisão definida de qual da dicotomia (Spec-Head-Comp e Comp-Head-Spec) seria mais razoável. Por isso, Wang (2005) propõe a ordem de complemento-núcleo-especificador para a análise da estrutura relativa em chinês. Wang (2005) propõe que, tendo em vista o estado sintático de "de" como C porposto por Ning (1993), “de" tem o traço wh- interpretável (Interpretable wh-feature) e o traço EPP forte ininterpretável (Strong uninterpretable EPP feature). Segundo Wang (2005), C “de” escolhe, na sua direção da esquerda, IP que contém núcleo da estrutura relativa como seu Comp como mostra (29a), e o núcleo se move da posição de objeto para a posição de Spec-CP que fica na direção da direita do C, como mostra (29a). Segundo 
Wang (2005), sua hipótese do núcleo C é útil para diferenciar estruturas relativas prenominais cujo núcleo $\mathrm{C}$ seja poteriorizado das estruturas relativas pós-nominais cujo núcleo $\mathrm{C}$ seja anteriorizado, apesar de não obedecer à ramificação binária do diagrama de árvore.

(29)a. [s-matriz wo xihuan] $\left[_{\mathrm{CP}}\right.$ IIP $_{\mathrm{IP}}$ nainai zuo $\left.\mathrm{t}_{\mathrm{i}}\right]$ de NPP $_{\mathrm{NP}}$ qiaokeli $\left.]_{\mathrm{i}}\right] . \quad$ (prenominal)

Eu adoro avó faz que chocolate

b. [s-matriz Eu adoro] $\left[_{\mathrm{CP}}\left[_{\mathrm{NP}}\right.\right.$ chocolate] que minha avó faz]. (pós-nominal)

[s-matriz I like] [ [CP [NP chocolate] that my grandma makes].

Such a proposal does not follow the popular idea that the binary branching of the tree diagram is absolutely uni-directional, instead, it allows for the practice of treating some categories as head-final ones while others as head-initial ones. (WANG, 2005, P.15)

Em resumo, diferentes análises (análise de núcleo externalizado, análise de promoção do núcleo e análise eclética) foram adotadas para a relativização em chinês, porém, as análises de Ning (1993), Aoun\&Li (2003) e Wang (2005) raramente examinam as evidências propostas por Chomsky $(1977,1981)$ para movimento, ou seja, esses estudo fazem um exelente trabalho para explicar a retivização em chinês, do ponto de vista descritivo. Segundo Chomsky, uma explicação adequada e plena para um fenômeno linguístico é uma explicação descritiva e explicativa. Para chegar ao nível explicativo desse fenômeno, a seção a seguir examinará se as evidências propostas por Chomsky como reconstrução, subjacência/efeitos de ilha e parasitic gap, são aplicadas à relativização em chinês, perante dados linguísticos concretos em chinês, para corroborar se a relativização em chinês é resultado de movimento.

\section{Evidências para a existência de estrutura relativa em chinês}

Segundo Chomsky (1977), certos aspectos sintáticos como, por exemplo, parsitic gaps, subjaência, efeitos de ilha, etc., têm que ser obedecidos para que haja movimento wh-.

The rule of wh-movement has the following general characteristics:

a. it leaves a gap.

b. where there is a bridge, there is an apparent violation of subjacency, the Propositional-Island Condition (PIC), and the Specified Subject Condition (SSC).

c. it observes the Complex Noun Phrase Constraint (CNPC).

d. it observes wh-island constraints.

(CHOMSKY, 1977, P. 86)

\subsection{Reconstrução}

Segundo Chomsky (1995), os efeitos de reconstrução (reconstruction effects) do reflexivo e da expressão idiomática é uma evidência importante para a existência de movimento do núcleo. No nível de representação, o reflexivo (herself)/ objeto da expressão idiomática (the headway) não está sob regência do seu antecedente (Maria)/ verbo da expressão idiomática como mostram (16) aqui repetida em (30) e (31). No nível de derivação, núcleo que contém reflexivo (the picture 
of herself)/ objeto da expressão idiomática (the headway) era regido por seu antecedente/verbo da expressão idiomática satisfazendo os princípios A e C propostos por Chomsky (1981), depois ele se move da posição de objeto da oração relativa CP como mostram (17) aqui repetida em (32) e (33).

(30) [DP the picture of himself $\left.{ }_{j}\right]_{i}\left[C_{C P}\right.$ that John ${ }_{j}$ painted $\left.t_{i}\right]$

(ALEXIADOU et al, 2000, P.7)

(31) [DP the headway $]_{i}\left[_{C P}\right.$ that we made $t_{i}$ was insufficient]

(ALEXIADOU et al, 2000, P.12)

(32) [DP $\mathrm{t}_{\mathrm{k}}$ ] [CP which [IP Maria ${ }_{\mathrm{j}}$ painted [DP the Picture of herself $\left.\mathrm{f}_{\mathrm{j}}\right]$ ]

(33) $\left[{ }_{D P} t_{k}\right] l_{C P}$ that [IP we made [DP the headway $\left.\left.]_{k}\right]\right]$

Pode-se observar que a derivação da estrutura relativa em inglês como resultado de movimento de núcleo permite à reconstrução de reflexivo e expressão idiomática, então, isso significa que se a derivação da estrutura em chinês permite também reconstrução, ela também é resultado de movimento. No nível de representação (34) e (36), o reflexivo (ziji)/ objeto (qi) da expressão idiomática (shengqi) $)^{9}$ não está sob regência do seu antecedente (dajia)/ verbo (sheng) da expressão idiomática como. No nível de derivação, núcleo que contém reflexivo (ziji de renwu)/ objeto (qi) da expressão idiomática era regido por seu antecedente/verbo da expressão idiomática satisfazendo os princípios A e C propostos por Chomsky (1981), depois ele se move da posição de objeto da oração relativa CP como mostram (35) e (37).

(34) $\left[_{\mathrm{CP}} \text { jingli rang dajia } \mathrm{k}_{\mathrm{k}} \text { yishidao } \mathrm{t}_{\mathrm{i}} \text { de] [DP } \text { ziji }_{\mathrm{k}} \text { de renwu }\right]_{\mathrm{i}}$

Gerente deixar a,gente ${ }^{10}$ conhecer $t_{\mathrm{i}}$ de se de meta (tradução literal)

a meta de cada um que o gerente deixou a gente conhecer (tradução adaptada)

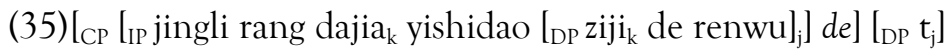

(36) [CP ta sheng $t_{\mathrm{i}}$ de] [DP qi $]_{\mathrm{i}}$ (rang tade laogong momingqimiao)

Ela irritar ti de irritação (deixar seu marido estranho) (tradução literal)

A irritação que ela tem (estranhou seu marido) (tradução adaptada)

(37) [CP [IP ta sheng [DP $\left.\mathrm{qi}_{\mathrm{k}}\right]$ de] [DP $\left.\mathrm{t}_{\mathrm{k}}\right]$ (rang tade laogong momingqimiao)

Assim, como a estrutura relativa em inglês, a em chinês também permite à reconstrução, então, é plausível dizer que ela é também resultado de movimento. Por isso, existe estrutura relativa em chinês não sendo como propõe a gramática tradicional.

\subsection{Subjacência/efeitos de ilha}

Segundo Chomsky (1977), uma das medidas importantes para determinar se uma estrutura relativa é resultado de movimento é examinar se ela obedece à subjacência/efeitos de ilha (subjacency/island effects) proposta inicialmente por Ross (1967) e Chomsky (1973). Segundo

\footnotetext{
9 "sheng qi" é uma expressão idiomática em chinês que significa "se irritar".

10 "a,gente" escrita dessa forma de é para adequar a forma "dajia” em chinês que é considerada um léxico único e que significa "a gente".
} 
Huang (1982), a estrutura relativa em inglês obedece à restrição de NP complexo (complex NP constraint) como mostra (a) e à restrição de sujeito como oração relativa (sentential subject constraint) como mostra (b), por isso, ela é resultado de movimento. Segundo Chomsky (1973), subjacência diz respeito ao fato de que não é permitido um movimento que ultrapasse dois nós cíclicos (cyclic bounding nodes) que sejam NP e S. A agramaticalidade de (a) e (b) se deve ao fato de que o movimento wh- (who) ultrapassa esse limite.

(a) * $\left[s{ }^{\prime}\right.$ who $_{i}\left[s\right.$ do you like [NP the books [s, that [s describe $\left.\left.\left.\left.t_{i}\right]\right]\right]\right]$ ?

(b) * [s' who $\mathrm{i}_{\mathrm{i}}\left[\mathrm{s}\right.$ did [NP [s' that [s she married $\left.\left.\left.\mathrm{t}_{\mathrm{i}}\right]\right]\right]$ surprise you]]?

Em (a), o operador wh- (who) se move do NP complexo para a posição de complemento da oração matriz. O movimento passa dois nós (bounding node) de S e um de NP, violando assim a subjacência... Em (b), a extração ( do operador wh- (who)) do sujeito como oração reltiva ultrapassa dois nós de S e um de NP...

Huang (1982, p. 456)

Do mesmo modo, se a estrutura relativa em chinês também obedece às duas restrições, ela é resultado de movimento. Diferente do que Huang (1982) e Ning (1993), que propõem a estrutura relativa em chinês é resultado de movimento de Op como propõe Chomsky (1977) para estrutura relativa em inglês que não contenha operador wh-, esse estudo propõe que ela é

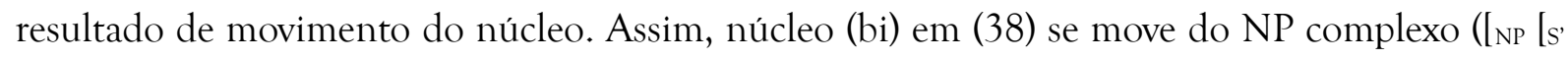
[s gei xuesheng $t_{k}$ ] de] nage ren]]]) para a posição de complemento da oração matriz ([s' [s ni zhidao [NP...] ma] bi $\mathrm{i}_{\mathrm{k}}$ ). A (38) fica agramatical, na medida em que o movimento passa dois nós de S (uma oração matriz e uma relativa) e um de NP (nage ren) violando subjacência. Da mesma maneira, núcleo (xin shu) em (39) se move do sujeito como oração relativa ([NP [s' [s Luxun xie $t_{i}$ ] de]]) para a posição de sujeito da oração matriz [s [NP ...] xiadao ta lema]). A (39) também fica agramatical dado que o movimento passa dois nós de S e um de NP violando também subjacência. Pode-se observar que a violação de restrição de NP complexo (38) e de restrição de sujeito como oração relativa (39) resulta da agramaticalidade, ou seja, a estrutura relativa em chinês obedece à subjacência. Por isso, ela é resultado de movimento.

$(38)^{*}\left[S^{\prime}\left[S\right.\right.$ ni zhidao $\left[{ }_{N P}\left[S^{\prime}\right.\right.$ [s gei xuesheng $\left.t_{k}\right]$ de $]$ nage ren]]] $\left.\left.\mathrm{ma}^{11}\right] \mathrm{bi}_{\mathrm{k}}\right]$ ?

Você sabe dar estudante $t_{k}$ que aquela pessoa canetas $s_{k}$

*[S what $_{k}\left[s\right.$ do you know [NP the person [S, who [s gave the students $\left.\left.\left.\left.t_{k}\right]\right]\right]\right]$ ?

$(39)^{*}\left[s^{\prime}\right.$ [s [NP [s' [s Luxun xie $\left.t_{i}\right]$ de] xiadao ta lema ${ }^{12}$ ] xin $s^{\prime} u_{i}$ ?

Luxun escrever $t_{i}$ que surpreender ele novo livro ${ }_{i}$ (tradução literal)

${ }^{*}\left[s\right.$, what ${ }_{i}\left[s\right.$ did [NP [s, that [s Luxun wrote $\left.\left.\left.t_{i}\right]\right]\right]$ surprise youl]?

\subsection{Parasitic gap}

Segundo Chomsky (1977), uma outra das medidas importantes de determinar se uma estrutura relativa é resultado de movimento é ver se ela permite parasitic gap (pg) ou não, se ela

\footnotetext{
${ }^{11}$ marca de questão sim-não (Yes-no question) no presente como do em inglês.

${ }^{12}$ marca de questão sim-não no passado como did em inglês.
} 
permite, é resultado de movimento. Estrutura relativa em inglês permite pg, por isso é resultado de movimento. Observe-se há duas categorias vazias (vestígio e pg) em (a), (b) e (c) sendo aquela que está na oração relativa matriz o vestígio e aquela que está na oração subordinada o pg. Além disso, há uma co-indexação entre núcleo (the students/the man/the professor), vestígio $\left(t_{i} / t_{k} / t_{j}\right)$ e pg $\left(\mathrm{pg}_{\mathrm{i}} / \mathrm{pg}_{\mathrm{k}} / \mathrm{pg}_{\mathrm{j}}\right)$ em (a), (b) e (c), dado que a permissão de stranding do verbo/sintagma verbal de forte transitividade (strong transitive (verb/verbal structure) stranding) (come and see/vote for/run into).

(a) The students ${ }_{i}$ that you persuade $t_{i}$ to invite us to come and see $\mathrm{pg}_{\mathrm{i}}$

(b) The man ${ }_{k}$ that you who are going to vote for $\mathrm{pg}_{\mathrm{k}}$ tell $\mathrm{t}_{\mathrm{k}}$

(c) The professor ${ }_{j}$ that you must say hello to $t_{j}$ if you run into $\mathrm{pg}_{\mathrm{j}}$

(ENGDAHL, 1983, p.11)

Pode-se ver que há principalmente três evidências, que são existência de duas categorias vazias (vestígio e pg) e de co-indexação entre núcleo, vetígio e pg e a permissão de stranding do verbo/sintagma verbal de forte transitividade, para provar a permissão de pg na estrutura relativa em inglês, subsequentemente, a existência de movimento. Então, se a estrutura relativa em chinês também se apresentar essas três evidências, pode-se dizer que ela também permite pg e é resultado de movimento. Em (40), existem duas categorias vazias (vestígio e pg) sendo aquela que está na oração relativa matriz ([CP IP $_{j} e_{j}$ kanguo $\left.t_{i}\right]$ de] [DP nabu dianying $\left.]_{i}\right)$ o vestígio e aquela que está na oração relativa inserida ([CP [IP $\mathrm{t}_{\mathrm{j}}$ dougei $\mathrm{pg}_{\mathrm{i}}$ hen gao pingjia ] de] [DP guanzhong $]_{\mathrm{j}}$ ) o pg. Além disso, há também uma co-indexação entre núcleo (nabu dianying), vestígio $\left(\mathrm{t}_{\mathrm{i}}\right)$ e pg $\left(\mathrm{pg}_{\mathrm{i}}\right)$, dado que a permissão de stranding do sintagma verbal de forte transitividade (gei something somebody/something). As três condições são satisfeitas, por isso, a estrutura relativa em chinês (40) permite pg e é resultado de movimento.

(40) kanguo $\mathrm{t}_{\mathrm{i}}$ de guanzhong dou gei $\mathrm{pg}_{\mathrm{i}}$ hen gao pingjia de nabu dianying ${ }_{\mathrm{i}}$ assistir $t_{\mathrm{i}}$ de espectadores todos dar $\mathrm{pg}_{\mathrm{i}}$ muito alta avaliação de aquele filme (tradução literal)

aquele filme que tem muito alta avaliação dos espectadores todos que o assistiram (tradução correta adaptada)

Se é plausível dizer que (40) permite pg, é controverso dizer que (41) também permite pg, na medida e que esse pg não é gerado por stranding do verbo/sintagma verbal de forte transitividade, ela está na posição de sujeito. Observe-se há também duas categorias vazias (vestígio e pg) em (41) sendo aquela que está na oração relativa matriz ([CP [IP $t_{i}$ chiguo] de] [DP naxie keren $]_{i}$ ) o vestígio e aquela que está na oração relativa inserida ([CP I $_{\text {IP }} \mathrm{pg}_{\mathrm{i}}$ dou kua $\mathrm{e}_{\mathrm{k}}$ hao] de] [DP naxie keren $]_{i}$ ) o pg. Além disso, há uma co-indexação entre núcleo (naxie keren), vestígio (ti) e pg (pgi), porém, essa co-indexação não é dada por stranding do verbo/sintagma verbal de forte transitividade. Ela é lançada por propriedade de permissão de sujeito nulo. Esse estudo propõe que a propriedade de sujeito nulo, além de verbo/sintagma verbal de forte transitividade, também permite pg e subsequentemente a estrutura relativa com propriedade de sujeito nulo como a em chinês é resultado de movimento.

(41) $t_{i}$ chiguo de $\mathrm{pg}_{\mathrm{i}}$ dou kua $\mathrm{e}_{\mathrm{k}}$ hao de naxie keren ${ }_{\mathrm{i}}$

$t_{i}$ experimentar de $\mathrm{pg}_{\mathrm{i}}$ todos elogiar $\mathrm{e}_{\mathrm{k}}$ bom de aqueles clientes ${ }_{\mathrm{i}}$ (tradução literal) 
Aqueles clientes que experimentaram X e que todos elogiaram X bom (tradução correta adaptada)

\section{Conclusão}

As estruturas modificador-núcleo propostas por gramáticos da gramática tradicional são especiais em termos de sua função sintática idêntica ao seu núcleo e de sua categorização como prototípica e atípica. O fato de modificador $(\mathrm{CP})$ ficar anterior do núcleo que é muito diferente para línguas como inglês e português em que CP fica posterior do núcleo e a existência de estruturas atributivas tradicionais e típicas de substantivo e de adjetivo que permitem a apagabilidade de de parecem nos dizer que realmente não existe estrutura relativa em chinês. No entanto, a estrutura atributiva verbal que não permite apagar de não parece uma estrutura atributiva real, o que a deixa especial, ou seja, ela é de fato uma estrutura relativa.

Fora do escopo da gramática tradicional, a derivação da estrutura relativa sempre é um assunto muito discutido no modelo gerativo. Chomsky (1977) propõe que estrutura relativa é resultado de operador wh- ou Op. Contudo, quando o núcleo for com reflexivo ou for objeto da expressão idiomática, essa proposta não dá conta de forma satisfatória. Para tratar melhor desse problema, Schachter (1973), Kayne (1994) e Vries (2002) propõem a análise de promoção do núcleo para a derivação da estrutura relativa. Resolvido esse problema, no entanto, a análise de promoção do núcleo aparentemente não consegue derivar adequadamente a estrutura relativa de adjunto. Aoun\&Li (2003) propõem que ela é resultado movimento de PP ou Op, sendo o núcleo gerado na base.

Com base nas diferentes análises (análise de núcleo externalizado, análise de promoção do núcleo e análise eclética) acima apresentadas, muitos linguistas têm explorado a derivação da estrutura reltiva em chinês, entre eles os estudos de Ning (1993), Aoun\&Li (2003) e Wang (2005) destacaram. Os trabalhos deles são exelentes do ponto de vista descritivo do fenêmeno, deixando uma lieratura preciosa para estudos futuros quanto à derivação da estrutura relativa em chinês. Porém, as evidências de movimento propostas por Chomsky $(1977,1981)$ são raramente examinadas.

Segundo Chomsky, uma explicação adequada e plena para um fenômeno linguístico é uma explicação descritiva e explicativa. Para chegar ao nível explicativo, esses estudo examinou as evidências propostas por Chomsky $(1977,1981)$ para movimento, como reconstrução, subjacência/efeitos de ilha e parasitic gap, para a relativização em chinês, contra aos dados linguísticos concretos. $\mathrm{O}$ resultado mostrou que as evidências se aplicam também para a relativização em chinês, por tanto, é resultado de movimento.

\section{Referências}

AlEXIADOU, A.; LAW, P.; MEINUNGER, A. \& WILDER, C. The Syntax of Relative Clauses. Amsterdam: John Benjamins, 2000.

AOUN, J.; LI, Y. A. Essays on the Representational and Derivational Nature of Grammar: The 
Diversity of WH-constructions. Cambridge, MA.: The MIT Press, 2003.

CHOMSKY, N. Conditions on transformations. In: ANDERSON, S.; KIPARSKY, P. (eds.). A Festschrift for Morris Halle, Holt, Rinehart, and Winston, New York, 1973.

CHOMSKY, N. On wh-movement. In: CULICOVE, P.; WASOW, T.; AKMAJIAN, A. (eds.). Formal Syntax. New York: Academic Press, 1977.

CHOMSKY, N. Lectures on Government and Binding. Dordrecht: Foris, 1981.

CHOMSKY, N. The Minimalist Program. Cambridge: MIT Press, 1995.

ENGDAHL, E. Parasitic gaps. Linguistic and Philosophy 6: pp. 5-34, 1983.

HUANG, J. Logical Relations in Chinese and the Theory of Grammar. New York: Garland, 1982.

KAYNE, R. The Antisymmetry of Syntax. Cambridge: MIT Press, 1994.

NING, C. Y. The Overt Syntax of Relativization and Topicalization in Chinese. University of California, Irvine, 1993.

ROSS, J. R. Constraints on Variables in Syntax. MIT PhD dissertation, 1967.

SCHACHTER, P. Focus and relativization. Language 49, pp. 19-46, 1973.

VRIES, M. The Syntax of Relativization. University of Amsterdam, Amsterdam, 2002.

WANG, Q. Derivation of Chinese Restrictive Relative Clauses. Hunan University, Changsha, 2005.

ZHANG, A. M.; HAN, L. Pianzheng jiegou de jvfa diwei. Journal of Jiangsu Normal University, Philosophy and Social Sciences Edition, 1997.

ZHOU, B. A Study on the "PP+VP" Phrase on Contemporary Chinese. Capital Normal University, Beijing, 2009.

Recebido em: 20/09/2019

Aceito em: 19/01/2020 